\title{
Pancreatin-Cetyl Pyridinium Chloride Digestion and Decontamination Method; A Novel, Sensitive, Cost-Effective Method for Culturing Mycobacterium tuberculosis
}

\author{
Pottathil Shinu ${ }^{1,2, *(\mathbb{D})}$, Anroop B. Nair ${ }^{3} \mathbb{D}$, Snawar Hussain ${ }^{1}$, Mohamed A. Morsy ${ }^{3,4}$ and Wafaa E. Soliman ${ }^{1,5} \mathbb{D}$ \\ 1 Department of Biomedical Sciences, College of Clinical Pharmacy, King Faisal University, \\ Al-Ahsa 31982, Saudi Arabia; snawar76@gmail.com (S.H.); weahmed@kfu.edu.sa (W.E.S.) \\ 2 Department of Microbiology, M.M. Institute of Medical Sciences and Research, \\ Maharishi Markandeshwar (Deemed to Be University), Mullana-Ambala 133207, India \\ 3 Department of Pharmaceutical Sciences, College of Clinical Pharmacy, King Faisal University, \\ Al-Ahsa 31982, Saudi Arabia; anair@kfu.edu.sa (A.B.N.); momorsy@kfu.edu.sa (M.A.M.) \\ 4 Department of Pharmacology, Faculty of Medicine, Minia University, El-Minia 61511, Egypt \\ 5 Department of Microbiology and Immunology, Faculty of Pharmacy, Delta University for Science \\ and Technology, Mansoura 11152, Egypt \\ * Correspondence: spottathail@kfu.edu.sa; Tel.: +966-551732794
}

Citation: Shinu, P.; Nair, A.B.;

Hussain, S.; Morsy, M.A.;

Soliman, W.E. Pancreatin-Cetyl Pyridinium Chloride Digestion and Decontamination Method; A Novel, Sensitive, Cost-Effective Method for Culturing Mycobacterium tuberculosis. Microorganisms 2021, 9, 2025. https:// doi.org/10.3390/

microorganisms 9102025

\section{Academic Editors:}

Isobella Honeyborne and

Giovanni Satta

Received: 23 August 2021

Accepted: 22 September 2021

Published: 24 September 2021

Publisher's Note: MDPI stays neutral with regard to jurisdictional claims in published maps and institutional affiliations.

Copyright: (c) 2021 by the authors. Licensee MDPI, Basel, Switzerland. This article is an open access article distributed under the terms and conditions of the Creative Commons Attribution (CC BY) license (https:/ / creativecommons.org/licenses/by/ $4.0 /)$.

\begin{abstract}
The present study evaluated the performance of newly developed pancreatin-cetylpyridinium chloride (pancreatin-CPC) digestion and decontamination method (DDM) with N-acetyl L-Cysteinesodium hydroxide (NALC-NaOH) DDM for isolation of Mycobacteria from clinically suspected pulmonary tuberculosis (PTB) patients. For the study, sputum samples $(n=613)$ obtained from clinically suspected PTB cases were subjected to direct microscopy, pretreatment with NALC-NaOH DDM (reference method), and pancreatin-CPC DDM followed by culture, and the data were analyzed. The direct microscopy illustrated diagnostic accuracies of $60.4 \%$ (sensitivity), $99.77 \%$ (specificity), 98.9\% (positive predictive value) and $88.3 \%$ (negative predictive value), respectively (against culture) for the detection of Mycobacterial species. The pancreatin-CPC DDM showed competitive diagnostic accuracies (against NALC-NaOH DDM) of 99.32\% (sensitivity), 94.07\% (specificity), 85.05\% (positive predictive value), and $99.76 \%$ (negative predictive value), respectively, for the isolation of Mycobacterial species. In conclusion, pancreatin-CPC DMM was a highly sensitive, technically simple, and cost-effective method, suggesting its competence to substitute the currently used NALC$\mathrm{NaOH}$ DDM.
\end{abstract}

Keywords: pulmonary tuberculosis; pancreatin digestion and decontamination method; non-tuberculous mycobacteria; Mycobacterium tuberculosis

\section{Introduction}

Tuberculosis (TB) continues to be a notable health menace across the globe despite effective treatment strategies endorsed by the World Health Organization [1]. Further, early detection and isolation of Mycobacterium tuberculosis (MTB) in culture is necessary for accurate detection of tuberculosis (TB). However, isolation of MTB and other nontuberculous mycobacteria (NTM) are hindered due to the presence of microbial flora in the patients' samples resulting in delayed confirmation of tubercle bacilli [2,3]. Currently, N-Acetyl L Cysteine (NALC)-sodium hydroxide $(\mathrm{NaOH})$ is the extensively used pretreatment technique to isolate MTB from clinical specimens. However, the literature indicates that pre-treatment of clinical samples with $\mathrm{NaOH}$ may kill a significant number of tubercle bacilli along with other contaminating organisms $[2,4,5]$.

Several pre-treatment methods have been investigated to isolate mycobacteria from sputum samples [2,4-11]. However, the complexity and low diagnostic accuracy of these methods limited their use in mycobacterial laboratories [6,9]. This endorses the significance 
of extensive research to develop highly sensitive and technically apparent methods for culturing MTB from clinical samples. This study investigated the performance of pancreatincetylpyridinium chloride (pancreatin-CPC DDM) with NALC-NaOH DDM to isolate TB bacilli from sputum samples obtained from clinically suspected pulmonary tuberculosis (PTB) patients. The NALC-NaOH DDM possesses various limitations such as being bulky, technically complex, the toxicity of $\mathrm{NaOH}$ against few strains of MTB, and instability of NALC solution (stable for $24 \mathrm{~h}$ once prepared). However, these problems can be overcome using pancreatin-CPC DDM, which is technically simple, easy to perform, nontoxicity of CPC against MTB strains, and stability of pancreatin-CPC solution (shelf life of one week once constituted. In the pancreatin- CPC DDM, pancreatin utilizes the property of mucolytics (for the lysis of sputum samples), and the other act as a disinfectant. Pancreatin, which is a mixture of various types of digestive enzymes (secreted by exocrine cells of the pancreas) and is capable of lysing carbohydrates, lipids, and proteins as well [12]. CPC, a mild disinfectant, which is specifically active against gram-positive bacteria [6]. The mucolytic property of pancreatin and disinfecting capacity of CPC is utilized in the pancreatin-CPC DDM. Therefore, this evaluation was aimed to compare the performance of two DDMs in the detection of tubercle bacilli from sputum samples.

\section{Materials and Methods}

\subsection{Study Setting}

A total of 613 consecutive sputa [two sputa (spot and morning) samples per patient] were obtained from suspected cases of PTB patients, who were attending the Revised National Tuberculosis Control Program (RNTCP) testing center at M. M. Institute of Medical Sciences, Ambala (India). The current study included all patients, who presented with a productive cough that lasts longer than two weeks or more or the patients as instructed by the physician. However, patients who were undergoing anti-tubercular treatment (ATT) were excluded from the study. All the sputum samples used in the study were obtained for direct Acid-fast bacilli (AFB) staining at the RNTCP center. AFB smear reports were distributed to the patients after the microscopic examination. Further, all the sputum was refrigerated overnight at $4-8{ }^{\circ} \mathrm{C}$ until used. Informed consent of the patients was obtained before sputum collection. After dispatching the AFB smear report, all the sputum samples were transported to the Department of Microbiology, M. M. Institute of Medical Sciences and Research, Ambala (India) for culture.

\subsection{Laboratory Methods}

\subsubsection{Homogenization of Sputum Samples}

Both sputum specimens (morning and spot) collected from each patient were pooled to obtain sufficient quantity $(4-6 \mathrm{~mL})$ and similar quality. These mixed sputum samples were homogenized for one min using a disposable Pasteur pipette followed by vortexing of a $3 \mathrm{~min}$ duration to assure the equal distribution of mycobacteria. All the procedures involving specimen segregation, smear preparation, and culture were carried out in a biological safety cabinet (Class II A) (Sterile Tech, Chennai, India). This homogenized sputum specimen was separated into three aliquots, namely; direct Auramine ' $\mathrm{O}$ ' staining and other two aliquots used for culture by NALC-NaOH DDM and pancreatin-CPC DDM.

\subsubsection{Direct Smear Microscopy}

For the light-emitting diode fluorescent microscopy (LED FM), direct Auramine ' $\mathrm{O}$ ' smears were prepared using a $0.5 \mathrm{~mL}$ homogenized sputum sample on a clean glass slide. Two independent technicians under 400 $\times$ magnification (Primo Star iLED, Carl Zeiss, Gottingen, Germany) microscopically examined the stained direct smears. The smear results were reported as per the guidelines published by RNTCP [13]. To measure the inter-observer variability, both technicians were blinded to each other's microscopic results when examining the direct AFB smears. To check the inter-observer variability, all the 
positive slides $(n=91)$ and $10 \%$ of randomly picked negative slides $(n=60)$ were examined by the study supervisor.

\subsubsection{NALC-NaOH DDM}

The second aliquot of homogenized sputum $(2 \mathrm{~mL})$ was subjected to NALC-NaOH DDM as described by Kent \& Kubica with minor changes (final $\mathrm{NaOH}$ concentration of $1 \%$ ) [14]. After the NALC-NaOH DDM procedure, $0.2 \mathrm{~mL}$ of the resulting pellet was cultured on Löwenstein-Jensen (LJ) media (two tubes, namely; one LJ tube containing sodium pyruvate and the other tube with glycerol). Each cultured LJ tubes was monitored weekly once during the eight weeks of aerobic incubation (at $37^{\circ} \mathrm{C}$ ).

\subsubsection{Pancreatin-CPC DDM}

The homogenized sputum sample $(2 \mathrm{~mL})$ was transferred to Falcon tube $(14 \mathrm{~mL}$ capacity) (BD Biosciences, San Jose, USA), added with an equal quantity of $4 \%$ pancreatin (Himedia, Mumbai, India) $-4 \%$ CPC (Himedia, Mumbai, India) working solution. This working solution was prepared by mixing an equal quantity of $4 \%$ pancreatin and $4 \%$ CPC stock solution, which was prepared in distilled water (DW)]. Then this reaction tube was subjected to vortexing $(1 \mathrm{~min})$ followed by $30 \mathrm{~min}$ incubation (at $37^{\circ} \mathrm{C}$ ). After incubation, DW was added and volume brought until the brim of tube followed by thorough mixing by vortexing and inversion. Later, this tube was centrifuged $(3300 \times g)$ for $20 \mathrm{~min}$ and sediment was separated after decanting the supernatant. The sediment was re-suspended with $200 \mu \mathrm{L}$ of DW and the resuspended pellet $(0.2 \mathrm{~mL})$ was cultured on two LJ culture tubes (one media with sodium pyruvate and the other with glycerol). These LJ slants were incubated (aerobically) at $37^{\circ} \mathrm{C}$ and checked for growth once every seven days for up to eight weeks.

\subsection{Identification of Mycobacteria Cultures}

The growth of mycobacteria was presumptively identified as per guidelines published by WHO [15]. Quality control of LJ slants was achieved using control strains [M. tuberculosis (ATCC H37Rv) and M. kansasii (ATCC 12478)]. Polymerase chain reaction (PCR) (primer sequence specific for 'IS6110') was used to confirm the MTB isolates. The primer sequence used includes; T4- 5'-CCT GCG AGC GTA GGC GTC GG $3^{\prime}$ and T5-5' CTC GTC CAG CGC CGC TTC GG $3^{\prime}$ with an expected band size of 123 bps [16].

\subsection{Statistical Analysis}

Diagnostic accuracies of NALC-NaOH DDM (reference methods) were calculated and compared against pancreatin-CPC DDM. The significance of differences in the sensitivities of NALC-NaOH DDM and pancreatin-CPC DDM were assessed using McNemar's test. The significance of differences in the rate of detection of mycobacteria was analyzed using Fisher's exact test carried out by GraphPad Prism 6 (Graph-Pad Software, Inc., La Jolla, CA, USA).

\section{Results}

Of 613 sputum specimens processed, direct AFB smear could detect a total of $14.85 \%$ $(91 / 613)$ smear-positive cases. However, LJ detected the presence of MTB and NTM in $22.83 \%(140 / 613)$ and $1.63 \%(10 / 613)$ of the specimens, respectively when treated with NALC-NaOH DDM (reference method). In contrast, sputum samples when digested and decontaminated using pancreatin-CPC DDM, the culture positivity of MTB and NTM were $25.77 \%$ (158/613) and that of NTM 2.61\% (16/613), respectively. Figure 1 depicts the study profile. Table 1 shows overall results (direct microscopy and culture) obtained after pretreatment with NALC-NaOH and pancreatin-CPC DDMs. Further, 2.28\% (14/613) and $3.1 \%(19 / 613)$ of specimens were contaminated in LJ culture after pretreatment with NALC-NaOH DDM and pancreatin-CPC DDM, respectively. After excluding the $4.07 \%$ $(25 / 613)$ of contaminated culture (obtained after pretreatment with all the methods studied), 
the culture positivity was found to be $23.64 \%$ (139/588) and 1.7\% (10/588) for MTB and NTM infections, respectively (after pretreatment with NALC-NaOH DDM). Similarly, after excluding $4.07 \%(25 / 613)$ contaminated culture results, $26.87 \%(158 / 588)$ and $2.72 \%$ $(16 / 588)$ patients were confirmed with MTB and NTM infections (in culture), respectively (after pretreatment with pancreatin-CPC DDM).

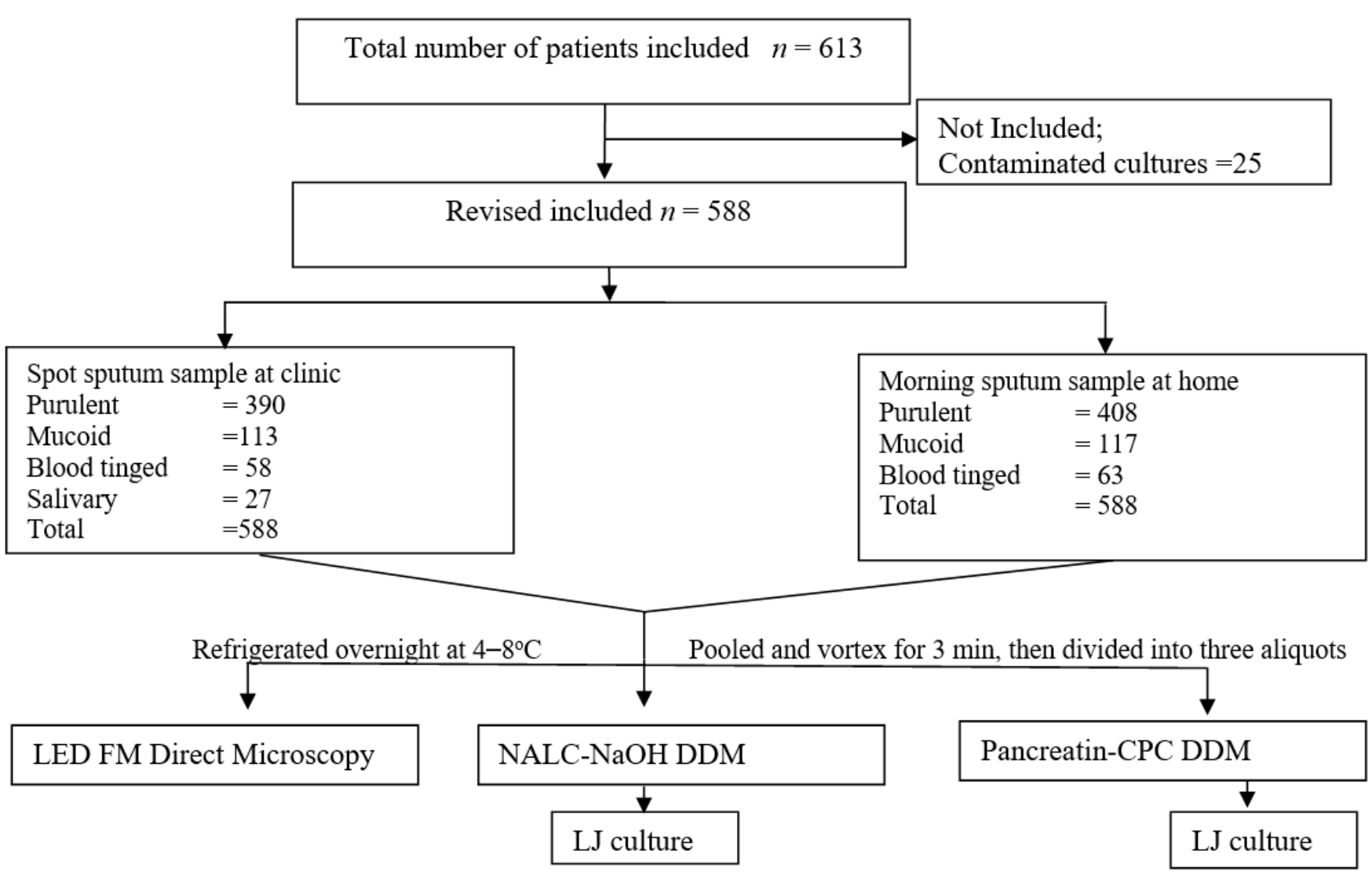

Figure 1. Flow chart showing study profile.

Table 1. Overall results (direct microscopy and culture results) were obtained after digestion and decontamination with NALC-NaOH (n-Acetyl-L-Cysteine-Sodium hydroxide) and Pancreatin-CPC (Cetyl Pyridinium Chloride) DDMs.

\begin{tabular}{|c|c|c|c|c|}
\hline \multirow{3}{*}{ Culture Results } & \multicolumn{2}{|c|}{ Direct LED FM Smear Positive Cases } & \multicolumn{2}{|c|}{ Total Samples Processed } \\
\hline & NALC-NaOH DDM & Pancreatin-CPC DDM & NALC-NaOH DDM & Pancreatin-CPC DDM \\
\hline & $\begin{array}{l}\text { LJ Culture } \\
(n=91) \%\end{array}$ & $\begin{array}{l}\text { LJ Culture } \\
(n=91) \%\end{array}$ & $\begin{array}{l}\text { LJ Culture } \\
(n=613) \%\end{array}$ & $\begin{array}{l}\text { LJ Culture } \\
(n=613) \%\end{array}$ \\
\hline a MTB & $85(93.4)$ & $86(94.5)$ & $140(22.83)$ & $158(25.77)$ \\
\hline${ }^{\mathrm{b}}$ NTM & $5(5.49)$ & $5(5.49)$ & $10(1.63)$ & $16(2.61)$ \\
\hline No growth & 1(1.09) & - & $457(74.55)$ & $428(69.82)$ \\
\hline Contaminated cultures & - & - & $14(2.28)$ & $19(3.1)$ \\
\hline Bacterial & - & - & $9(1.47)$ & $13(2.12)$ \\
\hline Fungal & - & - & - & $2(0.33)$ \\
\hline Liquefied LJ media & - & - & $5(0.82)$ & $4(0.65)$ \\
\hline
\end{tabular}

${ }^{a}$ Mycobacterium tuberculosis; ${ }^{\text {b }}$ Non tuberculosis mycobacteria; LJ, Löwenstein-Jensen.

Table 2 shows the comparison of isolation rates of MTB and NTM using LJ culture after pretreatment with alkali and CPC-based DDMs as demonstrated by LED-FM smear scores and quality of sputum. Of the total smear-positive sputum samples $(n=91), 60.4 \%(90 / 149)$ of mycobacterial isolates were grown in LJ culture after treatment with alkali-based NALC- 
$\mathrm{NaOH}$ DDM (Table 2). Table 2 illustrates that LJ culture (LJ, $n=174$, after treatment with pancreatin-CPC DDM) detected twenty-five additional mycobacterial isolates in comparison with alkali-based DDM (LJ, $n=149$ ) (Fisher's exact test, $p<0.05$ ). Table 3 shows the culture results yielded after digestion and decontamination with NALC-NaOH and pancreatin-CPC DDM as shown by LED-FM direct microscopy. Interestingly, a direct relationship between direct LED FM smear scores and culture yield was noted. Of the total MTB isolates yielded after treatment with alkali-based $(n=149)$, and CPC $(n=174)$ based DDMs, $29.53 \%$ (44/149) and 27\% (47/174) sputum samples were having $3+$ and $2+$ smear scores. These samples also showed heavy growth (3+ and 2+ growth) in LJ culture (Table 3). In contrast, the majority of the sputum samples with low bacillary load (such as sputum samples with $1+$ and scanty smear scores) showed comparatively fewer numbers of mycobacterial colonies in culture.

Table 4 shows the growth detection time of LJ culture for recovery of MTB and NTM after treatment with alkali and CPC-based DDMs as indicated by direct microscopy. Table 4 depicts that most of the sputum samples possessing $3+$ and $2+$ smear grades showed growth of tubercle bacilli within 2-4 weeks (LJ, $n=40$ ), irrespective of the type of DDMs used. Alternatively, delayed growth of MTB was noted in samples with 1+ or scanty smear grades. Nevertheless, the differences between the meantime to detection of MTB (on LJ media) for CPC-based DDM was 36.23 days (SD 10.06), which was relatively less when compared to alkali-based DDM (38.3 days; SD 9.72). Likewise, LJ culture showed growth of NTM with a mean detection time of 20.4 (SD 3.29) and 15.94 days (SD 3.5) for samples treated with alkali-based DDM and CPC-based DDM, respectively. Table 5 demonstrates sensitivity and specificity of $99.32 \%$ and $94 \%$ for LJ culture (when specimens treated with pancreatin-CPC DDM) in comparison with alkali-based DDM (McNemar's test, $p<0.0001$ ) to isolate mycobacterial species (Table 5). 


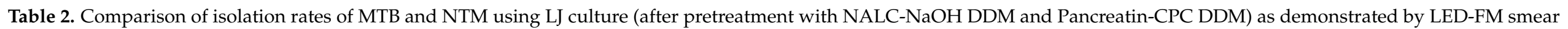
scores and quality of sputum.

\begin{tabular}{|c|c|c|c|c|c|c|c|c|c|c|c|c|c|c|c|c|c|c|c|c|c|c|c|}
\hline \multicolumn{13}{|c|}{ NALC-NaOH Digestion and Decontamination Methods } & \multicolumn{11}{|c|}{ Pancreatin-CPC Digestion and Decontamination Methods } \\
\hline \multicolumn{13}{|c|}{ LJ Culture } & \multicolumn{11}{|c|}{ LJ Culture } \\
\hline \multirow[b]{2}{*}{ Quality of sputum } & \multicolumn{12}{|c|}{ * LED FM smear score } & \multicolumn{11}{|c|}{ * LED FM smear score } \\
\hline & \multicolumn{2}{|c|}{$3+(n=34)$} & \multicolumn{2}{|c|}{$2+(n=18)$} & \multicolumn{2}{|c|}{$1+(n=25)$} & \multicolumn{3}{|c|}{ Scanty $(n=14)$} & \multicolumn{3}{|c|}{$\begin{array}{c}\text { Negative } \\
\text { (N497) }\end{array}$} & \multicolumn{2}{|c|}{$3+(n=34)$} & \multicolumn{2}{|c|}{$2+(n=18)$} & \multicolumn{2}{|c|}{$1+(n=25)$} & \multicolumn{2}{|c|}{ Scanty $(n=14)$} & \multicolumn{3}{|c|}{ Negative $(n=497)$} \\
\hline Purulent $(n=408)$ & 22 & - & 8 & 1 & 99 & 1 & 5 & 2 & - & 52 & 1 & 307 & 22 & - & 8 & 1 & 99 & 1 & 5 & 2 & 59 & 3 & 298 \\
\hline $\operatorname{Mucoid}(n=117)$ & 9 & - & 4 & - & 9 & - & 4 & - & - & 2 & 2 & 87 & 9 & - & 4 & - & 9 & & 4 & 1 & 8 & 2 & 81 \\
\hline $\begin{array}{l}\text { Blood tinged(63) } \\
\text { Subtotal }\end{array}$ & $\begin{array}{c}3 \\
34\end{array}$ & - & $\begin{array}{c}5 \\
17\end{array}$ & $\overline{1}$ & $\begin{array}{c}6 \\
24\end{array}$ & $\overline{1}$ & $\begin{array}{c}1 \\
10\end{array}$ & $\begin{array}{l}1 \\
3\end{array}$ & $\begin{array}{l}1 \\
1\end{array}$ & $\overline{54}$ & $\begin{array}{l}2 \\
5\end{array}$ & $\begin{array}{c}44 \\
438\end{array}$ & $\begin{array}{c}3 \\
34\end{array}$ & - & $\begin{array}{c}5 \\
17\end{array}$ & $\overline{1}$ & $\begin{array}{c}6 \\
24\end{array}$ & 1 & $\begin{array}{c}2 \\
11\end{array}$ & $\begin{array}{l}1 \\
3\end{array}$ & $\begin{array}{c}5 \\
72\end{array}$ & $\begin{array}{c}6 \\
11\end{array}$ & $\begin{array}{c}35 \\
414\end{array}$ \\
\hline Total number of culture isolates & \multicolumn{12}{|c|}{149} & \multicolumn{11}{|c|}{174} \\
\hline
\end{tabular}

* as per Revised National Tuberculosis Control Program; NG-No growth.

Table 3. Culture results yielded after digestion and decontamination with NALC-NaOH and Pancreatin CPC DDM as shown by LED-FM direct microscopy.

\begin{tabular}{|c|c|c|c|c|c|c|c|c|c|c|c|c|c|c|c|c|c|c|}
\hline \multirow{4}{*}{$\begin{array}{c}\text { Direct Smear } \\
\text { Scores } \\
\text { (LED FM) }\end{array}$} & \multicolumn{18}{|c|}{ Culture on LJ Media } \\
\hline & \multicolumn{9}{|c|}{ NALC-NaOH DDM } & \multicolumn{9}{|c|}{ Pancreatin-CPC DDM } \\
\hline & \multicolumn{2}{|c|}{$\begin{array}{c}\text { a } 3+ \\
(n=45 *)\end{array}$} & \multicolumn{2}{|c|}{$\begin{array}{c}\mathrm{b}^{2} 2+ \\
(n=28 *)\end{array}$} & \multicolumn{2}{|c|}{$\begin{array}{c}{ }^{\mathrm{c}} 1+ \\
(n=31 *)\end{array}$} & \multicolumn{2}{|c|}{$\begin{array}{l}\text { d Scanty } \\
\left(n=45^{*}\right)\end{array}$} & \multirow[t]{2}{*}{$\begin{array}{c}\text { NG } \\
(n=439)\end{array}$} & \multicolumn{2}{|c|}{$\begin{array}{c}\text { a } 3+ \\
(n=63 *)\end{array}$} & \multicolumn{2}{|c|}{$\begin{array}{c}\mathrm{b} 2+ \\
\left(n=31^{*}\right)\end{array}$} & \multicolumn{2}{|c|}{$\begin{array}{c}{ }^{\mathrm{c}} 1+ \\
(n=34 *)\end{array}$} & \multicolumn{2}{|c|}{$\begin{array}{l}\text { d Scanty } \\
\left(n=46^{*}\right)\end{array}$} & \multirow{2}{*}{$\begin{array}{c}\text { NG } \\
(n=414\end{array}$} \\
\hline & МТВ & NTM & МТВ & NTM & МТВ & NTM & МТВ & NTM & & МТВ & NTM & МТВ & NTM & МТВ & NTM & МТВ & NTM & \\
\hline $3+(n=34)$ & 19 & - & 12 & - & 2 & - & 1 & - & - & 24 & - & 7 & - & 3 & - & - & - & - \\
\hline $2+(n=18)$ & 7 & 1 & 5 & - & 4 & - & 1 & - & - & 11 & 1 & 4 & - & 2 & - & - & - & - \\
\hline $1+(n=25)$ & 6 & - & 1 & - & 7 & 1 & 10 & - & - & 8 & - & 5 & - & 7 & 1 & 4 & - & - \\
\hline Negative $(n=497)$ & 7 & 2 & 5 & 2 & 13 & 1 & 29 & - & 438 & 9 & 7 & 6 & 3 & 18 & 1 & 39 & - & 414 \\
\hline Total number of culture isolates & & & & 149 & & & & & & & & & & 174 & & & & \\
\hline
\end{tabular}

a 3+-more than 200 colonies; $^{\text {b }}$ 2+-100 to 200 colonies; $^{\mathrm{c}}$ 1+-20 to 99 colonies; ${ }^{\mathrm{d}}$ Scanty-less than 20 colonies; NG-No growth; ${ }^{*}$ indicates presence of non-tuberculous mycobacteria. 
Table 4. Growth detection time of LJ culture for recovery of Mycobacterium tuberculosis (MTB) and non-tuberculous Mycobacteria (NTM) after digestion and decontamination with NALC-NaOH and pancreatin-CPC DDM as indicated by LED-FM smear scores.

\begin{tabular}{|c|c|c|c|c|c|c|c|c|c|c|}
\hline \multirow{4}{*}{$\begin{array}{l}\text { Growth Detection Time } \\
\text { (Weeks) }\end{array}$} & \multicolumn{10}{|c|}{ LED-FM Direct Smear Scores and Culture Results } \\
\hline & \multicolumn{5}{|c|}{ NALC-NaOH DDM } & \multicolumn{5}{|c|}{ Pancreatin-CPC DDM } \\
\hline & \multicolumn{5}{|c|}{ LJ Culture } & \multicolumn{5}{|c|}{ LJ Culture } \\
\hline & $\begin{array}{c}3+ \\
n=34\end{array}$ & $\begin{array}{c}2+ \\
n=18\end{array}$ & $\begin{array}{c}1+ \\
n=25\end{array}$ & $\begin{array}{l}\text { Scanty } \\
n=13\end{array}$ & $\begin{array}{l}\text { Negative } \\
n=59\end{array}$ & $\begin{array}{c}3+ \\
n=34\end{array}$ & $\begin{array}{c}2+ \\
n=18\end{array}$ & $\begin{array}{c}1+ \\
n=25\end{array}$ & $\begin{array}{l}\text { Scanty } \\
n=14\end{array}$ & $\begin{array}{l}\text { Negative } \\
n=83\end{array}$ \\
\hline \multicolumn{11}{|c|}{ MTB } \\
\hline$\leq 1$ & - & - & - & - & - & & & & & \\
\hline 2 & 11 & 7 & 1 & - & 1 & 13 & 10 & 2 & - & 4 \\
\hline 3 & 12 & 5 & 1 & - & 6 & 9 & 2 & - & 1 & 11 \\
\hline 4 & 3 & 1 & 4 & 2 & 7 & 5 & 3 & 3 & - & 16 \\
\hline 5 & 5 & 3 & 6 & 1 & 2 & 3 & - & 7 & 3 & 10 \\
\hline 6 & 1 & 1 & 5 & 2 & 9 & 2 & 1 & 9 & 5 & 5 \\
\hline 7 & 2 & - & 5 & 3 & 12 & 2 & 1 & 2 & - & 19 \\
\hline 8 & - & - & 2 & 2 & 17 & - & - & 1 & 2 & 7 \\
\hline \multicolumn{11}{|c|}{ NTM } \\
\hline$\leq 1$ & - & - & - & 1 & - & - & 1 & 1 & - & 3 \\
\hline 2 & - & - & - & - & 1 & - & - & - & 1 & 2 \\
\hline 3 & - & 1 & 1 & - & 1 & - & - & - & 2 & 5 \\
\hline 4 & - & - & - & 2 & 3 & - & - & - & - & 1 \\
\hline 5 & - & - & - & - & - & - & - & - & - & \\
\hline 6 & - & - & - & - & - & - & - & - & - & \\
\hline 7 & - & - & - & - & & - & - & - & - & \\
\hline 8 & - & - & - & - & & - & - & - & - & \\
\hline
\end{tabular}

Table 5. Diagnostic accuracy of direct microscopy and culture for detection of Mycobacterium tuberculosis (MTB) and non-tuberculous Mycobacteria (NTM) after treatment with NALC-NaOH and pancreatin-CPC DDMs.

\begin{tabular}{|c|c|c|c|c|c|c|c|c|c|c|c|}
\hline \multirow{2}{*}{ Methods } & & \multicolumn{2}{|c|}{ Culture } & \multirow{2}{*}{$\begin{array}{c}\text { Sensitivity } \\
(\%)\end{array}$} & \multirow{2}{*}{ 95\% C.I. } & \multirow{2}{*}{$\begin{array}{l}\text { Specificity } \\
(\%)\end{array}$} & \multirow{2}{*}{ 95\% C.I. } & \multirow{2}{*}{$\begin{array}{c}\text { PPV } \\
(\%)\end{array}$} & \multirow{2}{*}{ 95\% C.I. } & \multirow{2}{*}{$\begin{array}{l}\text { NPV } \\
(\%)\end{array}$} & \multirow{2}{*}{$95 \%$ C.I. } \\
\hline & & Positive & Negative & & & & & & & & \\
\hline * Direct LED-FM smear & $\begin{array}{l}\text { Positive } \\
\text { Negative }\end{array}$ & $\begin{array}{l}90 \\
59\end{array}$ & $\begin{array}{c}1 \\
438\end{array}$ & 60.4 & $52.04-68.21$ & 99.77 & $98.53-99.98$ & 98.9 & $93.17-99.94$ & 88.3 & $84.88-90.78$ \\
\hline $\begin{array}{l}\text { Pancreatin-CPC DDM } \\
\quad * \text { LJ culture }\end{array}$ & $\begin{array}{l}\text { Positive } \\
\text { Negative }\end{array}$ & $\begin{array}{c}148 \\
1\end{array}$ & $\begin{array}{c}26 \\
413\end{array}$ & 99.32 & $95.75-99.96$ & 94.07 & $91.33-96$ & 85.05 & $78.68-89.83$ & 99.76 & $98.45-99.98$ \\
\hline
\end{tabular}

* Number of specimens positive for Mycobacterium tuberculosis and non-tuberculous mycobacteria $(n=149)$ and negative for mycobacteria $(n=429)$ after treatment with NALC-NaOH DDM for calculating diagnostic accuracy of LJ culture.

\section{Discussion}

Tuberculosis remains the foremost cause of death across the globe, particularly in Southeast Asian countries. Microbiological detection of TB is essential to stop the progression of TB [1]. Diagnosis of TB can be made by direct microscopic examination, culture, histological and radiological examinations [2]. However, culture is regarded as the gold standard to diagnose active TB. Further, effective isolation of MTB in culture depends on the type of DDMs used [2]. This study compared the functioning of NALC-NaOH DDM with newly developed pancreatin-CPC DDM. In the present study, patients who received ATT in the last six months were excluded as ATT may influence the viability of MTB in culture irrespective of the duration of treatment. The other characteristic of the current study design was the mixing of spot sputum samples with early morning sputum samples to enhance the quality of sputum; indeed, the majority of the samples collected at the hospital with a mucoid or salivary consistency, and when this sputum was pooled with morning sample, a purulent consistency was obtained.

In this study, culture detected the presence of MTB in $22.84 \%(140 / 613)$ of the specimens when treated with NALC-NaOH DDM in comparison with direct AFB smear $14.84 \%$ $(91 / 613)$. These results were consistent with earlier works as well, in which they inves- 
tigated various rapid methods for the detection of MTB in parallel with conventional LJ culture [2]. However, a higher detection rate of MTB was noted when specimens were treated with pancreatin-CPC DDM (detection rate of MTB was $25.77 \%$ ) signifying the impending nature of pancreatin-CPC DDM for the effective isolation of MTB than NALC-NaOH DDM. Further, of the total samples processed using NALC-NaOH DDM, NTM growth was observed in $1.63 \%$ of cases, which was comparable with earlier works published from this geographical location [17,18].

The isolation rate of NTM was considerably increased when sputum specimens were treated with pancreatin-CPC DDM (LJ, 2.61\%) suggesting the potential of pancreatin-CPC DDM for the effective isolation of NTM as well. In the current study, the rate of contamination was found to be low for sputum samples treated with NALC-NaOH $(1.8 \%)$ and pancreatin-CPC DDM (3.09\%) and the differences in the contamination rate were insignificant. This minimum rate of contamination of the NALC-NaOH decontaminated samples may be because of a low concentration of $\mathrm{NaOH}$. Nevertheless, the relatively low contamination rate of pancreatin-CPC DDM may be endorsed due to the bacteriostatic action of CPC, which is a quaternary ammonium compound that disrupts bacterial cell membranes (especially the commensal bacteria in the upper respiratory tract) and subsequently resulting in the death of bacteria.

LED FM continues to be the primary diagnostic tool for peripheral TB laboratories and peripheral health centers with limited resources [13]. In this study, LED FM documented sensitivity and specificity of $60.4 \%$ and $99.77 \%$, respectively. This microscopic technique revealed a diagnostic accuracy of $60.4 \%$ (sensitivity) and $99.77 \%$ (specificity), respectively. It was consistent with previous studies wherein the diagnostic accuracy for the LED FM ranged between 65-85\% and 80-90\%, respectively $[19,20]$. Further, direct LED microscopy detected one smear-positive but culture-negative case after treatment with the NALC-NaOH method. Conversely, the same cases were detected by LJ culture after treatment with pancreatin-CPC DDM. This culture-negative but smear-positive (false positive) results perhaps due to the death of tubercle bacilli caused by the alkaline toxicity that must have been induced during the NALC-NaOH DDM procedure [2]. However, culture possesses many advantages over LED FM, indeed; LJ culture detected additional cases of mycobacteria in $10 \%(59 / 588)$ and $14.12 \%(83 / 588)$ of sputum specimens when treated with NALC-NaOH and pancreatin-CPC DDMs, respectively. This increased detection rate of culture may be imputed to the diagnostic accuracy of culture, which is used to isolate tubercle bacilli particularly in samples with low bacillary load [21]. Culture exhibited an early growth of tubercle bacilli in most of the samples (LJ, $n=40$ ) that possess higher smear grades regardless of the pre-treatment method used (Table 4). However, the meantime to detection of bacillary growth was increased ( 6 weeks or more) in specimens having low smear grades (1+ and scanty). This can be explained based on smear scores; more specifically, smear scores depend on the number of bacilli present in the specimens. Therefore, specimens with higher smear scores, show reduced growth detection time.

Recently, various DDMs have been investigated and most of these DDMs use a chemical agent for digestion and the other as a disinfectant [2,4-10]. The most commonly used NALC-NaOH digestion and decontamination technique uses $0.5 \%$ NALC as a mucolytic agent and $\mathrm{NaOH}$ (final concentration of 1\%) as a decontaminating agent [14]. In the current study, culture positivity was relatively high exclusively for smear-negative samples (=59) after treatment with alkali-based DDM. This increased isolation rate of mycobacteria could be attributed to the improved sensitivity of culture, which is higher than that of microscopy. Further, culture detected growth of MTB in most of the samples (when treated with NALC$\mathrm{NaOH}$ DDM) and it was noted that all these strains were found to be grown in LJ culture after treatment with pancreatin-CPC DDM as well. This is probably due to the chemical properties of the various samples and their response to various pre-treatment procedures. The other limitations of NALC-NaOH DDM include (a) the duration of pretreatment of the specimen with alkali is limited, indeed; as the duration of alkaline treatment is increased, that may cause toxic effect on few strains MTB. Therefore, the duration of alkaline exposure 
is vital and limited to $20 \mathrm{~min}$. (b) need to adjust the $\mathrm{pH}$ of the NALC-NaOH DDM during the pre-treatment process.

In pancreatin-CPC DDM, pancreatin (4\%) and CPC (4\%) function as digestive, and decontaminating agents, respectively. The particular concentration of $4 \%$ pancreatin $-4 \%$ CPC used in this study was determined after conducting a pilot study using varying concentrations of pancreatin. It was noted that overnight treatment of clinical samples with $1 \%$ CPC could decontaminate the samples effectively [2]. However, the current study used a higher concentration of CPC ( $4 \%$ ) to minimize the duration of pretreatment (such as $30 \mathrm{~min}$ ). Considering these points in mind, we tested varying concentrations of pancreatin $0.5 \%, 1 \%, 2 \%$, $3 \%, 4 \%$ and $5 \%$ in combination with $4 \%$ CPC against AFB smear-positive sputum samples $(n=10)$. This reaction mixture was incubated at $37^{\circ} \mathrm{C}$ for $30 \mathrm{~min}$. The results of the pilot study suggested that $4 \%$ concentration of both pancreatin and CPC might be effective for isolation of MTB. In this study, culture detected a significant number of MTB isolates when smear-negative samples were treated with pancreatin-CPC DDM (LJ culture, $n=83$ ) than those specimens when exposed to NALC-NaOH DDM (LJ culture, $n=59$ ). The differences between these were found to be significant (Fisher's exact test, $p<0.05$ ). These increased false-negative results can be endorsed to the lytic property of pancreatin, which contains a mixture of lytic enzymes that could disintegrate the sputum samples to discharge the bacilli embedded in the mucus clumps and macrophages. It was also noted that the growth detection time was relatively reduced when specimens treated with pancreatin-CPC DDM as compared to those specimens that were treated with NALC-NaOH DDM (Table 4). This is possible may be due to the nontoxic action of CPC on the viability of mycobacterial species. This further suggests that pancreatin-CPC DDM may be used as an alternative method for NALC-NaOH DDM. Pancreatin-CPC DDM also possesses several advantages over NALC-NaOH DDM. This includes the stability of pancreatin-CPC solution (shelf life of one week once constituted), easy preparation, autoclaving of the pancreatin-CPC solution is not required as CPC itself a self-decontaminating solution, washing with distilled water is not required before the inoculation on LJ media, and thereby spares the time of technologists. The approximate cost for pancreatin-CPC and NALC-NaOH DDM was found to be $\sim 5$ ₹ and $\sim 10$ ₹, respectively. However, the pancreatin-CPC DDM also possesses few disadvantages like the possibility of crystal formation while the working solution is kept at a low temperature $\left(<22{ }^{\circ} \mathrm{C}\right)$ [6]. However, this problem can be resolved when these solutions are kept at a temperature $>25^{\circ} \mathrm{C}$. Another limitation of CPC-treated specimens includes a relatively low isolation rate of MTB when the CPC-treated specimens are cultured in $\mathrm{BBL}^{\mathrm{TM}} \mathrm{MGIT}^{\mathrm{TM}}$ tubes (as they may interfere with fluorescent signals of BBL MGIT) [22]. However, this negative effect of CPC-treated specimens can be minimized, when all the sediments obtained after treatment with pancreatin-CPC DMM may be washed with phosphate buffer before inoculation into BBL MGIT tubes.

\section{Conclusions}

In summary, LJ culture showed an incremental yield in the recovery of mycobacterial isolates mainly from smear-negative sputa that were treated with pancreatin-CPC DDM than NALC-NaOH DDM treated sputa, suggesting its promising use in clinical TB laboratories.

Author Contributions: Conceptualization, P.S., A.B.N., S.H.; methodology, P.S., A.B.N., M.A.M., S.H. and W.E.S.; formal analysis, P.S., A.B.N., M.A.M. and S.H.; resources, P.S.; writing-original draft preparation, P.S., A.B.N., M.A.M. and W.E.S.; writing-review and editing, P.S., A.B.N., M.A.M., S.H. and W.E.S.; project administration, P.S., A.B.N., M.A.M.; funding acquisition, P.S. All authors have read and agreed to the published version of the manuscript.

Funding: The authors acknowledge the Deanship of Scientific research at King Faisal University for the financial support under Nasher Track (Grant No. 216048). 
Institutional Review Board Statement: This study received ethical approval from Maharishi Markandeshwar University ethical committee (MMIMSR/IEC/2019/1456, dated: 20/04/2019) and informed consent was also collected from the patients.

Informed Consent Statement: Informed consent was obtained from all subjects involved in the study.

Data Availability Statement: All relevant data are provided in the manuscript.

Acknowledgments: The authors thank the laboratory technician Gulab Singh and Manoj Sharma for the technical support in the conduct of this study. The funders had no role in study design, data collection, and analysis, decision to publish, or preparation of the manuscript.

Conflicts of Interest: The authors declare that there is no conflict of interest.

\section{References}

1. World Health Organization (WHO). Global Tuberculosis Report. 2019. Available online: https://www.who.int/tb/publications/ global_report/en/ (accessed on 10 May 2021).

2. Shinu, P.; Singh, V.; Nair, A.; Mehrishi, P.; Mehta, S.; Joshi, E. Application of cetylpyridinium chloride and sodium chloride decontamination method for recovery of Mycobacterium tuberculosis from clinically suspected cases of pulmonary tuberculosis. Diagn. Microbiol. Infect. Dis. 2013, 77, 150-157. [CrossRef] [PubMed]

3. Miggiano, R.; Rizzi, M.; Ferraris, D.M. Mycobacterium tuberculosis Pathogenesis, infection prevention and treatment. Pathogens 2020, 9, 385. [CrossRef] [PubMed]

4. Ganoza, C.A.; Ricaldi, J.N.; Chauca, J.; Rojas, G.; Munayco, C.; Agapito, J.; Palomino, J.C.; Guerra, H. Novel hypertonic salinesodium hydroxide (HS-SH) method for decontamination and concentration of sputum samples for Mycobacterium tuberculosis microscopy and culture. J. Med. Microbiol. 2008, 57, 1094-1098. [CrossRef] [PubMed]

5. Shinu, P.; Singh, V.A.; Nair, A.; Venugopala, K.N.; Akrawi, S.H. Papain-cetylpyridinium chloride and pepsin-cetylpyridinium chloride; two novel, highly sensitive, concentration, digestion and decontamination techniques for culturing mycobacteria from clinically suspected pulmonary tuberculosis cases. PLoS ONE 2020, 15, e0236700. [CrossRef] [PubMed]

6. Smithwick, R.W.; Stratigos, C.B.; David, H.L. Use of cetylpyridinium chloride and sodium chloride for the decontamination of sputum specimens that are transported to the laboratory for the isolation of Mycobacterium tuberculosis. J. Clin. Microbiol. 1975, 1, 411-413. [CrossRef] [PubMed]

7. Chakravorty, S.; Tyagi, J.S. Novel multipurpose methodology for detection of mycobacteria in pulmonary and extrapulmonary specimens by smear microscopy, culture, and PCR. J. Clin. Microbiol. 2005, 43, 2697-2702. [CrossRef] [PubMed]

8. Pardini, M.; Varaine, F.; Iona, E.; Arzumanian, E.; Checchi, F.; Oggioni, M.R.; Orefici, G.; Fattorini, L. Cetyl-pyridinium chloride is useful for isolation of Mycobacterium tuberculosis from sputa subjected to long-term storage. J. Clin. Microbiol. 2005, 43, 442-444. [CrossRef] [PubMed]

9. Ricaldi, J.N.; Guerra, H. A simple and improved method for diagnosis of tuberculosis using hypertonic saline and sodium hydroxide to concentrate and decontaminate sputum. Trop. Dr. 2008, 38, 97-99. [CrossRef]

10. De Bel, A.; De Geyter, D.; De Schutter, I.; Mouton, C.; Wellemans, I.; Hanssens, L.; Schelstraete, P.; Malfroot, A.; Pierard, D. Sampling and decontamination method for culture of nontuberculous mycobacteria in respiratory samples of cystic fibrosis patients. J. Clin. Microbiol. 2013, 51, 4204-4206. [CrossRef] [PubMed]

11. Stephenson, D.; Perry, A.; Nelson, A.; Robb, A.E.; Thomas, M.F.; Bourke, S.J.; Perry, J.D.; Jones, A.L. Decontamination Strategies Used for AFB Culture Significantly Reduce the Viability of Mycobacterium abscessus Complex in Sputum Samples from Patients with Cystic Fibrosis. Microorganisms 2021, 9, 1597. [CrossRef] [PubMed]

12. Mojica, L.; Chen, K.; de Mejía, E.G. Impact of commercial precooking of common bean (Phaseolus vulgaris) on the generation of peptides, after pepsin-pancreatin hydrolysis, capable to inhibit dipeptidyl peptidase-IV. J. Food Sci. 2015, 80, H188-H198. [CrossRef] [PubMed]

13. Central TB Division; Directorate General of Health Services; Ministry of Health and Family Welfare. Revised National Tuberculosis Control Programme. In Manual for Laboratory Technicians; Central TB Division, Directorate General of Health Services, Ministry of Health and Family Welfare: New Delhi, India, 2012.

14. Kent, P.T.; Kubica, G.P. Public Health Mycobacteriology: A Guide for the Level III Laboratory; US Department of Health and Human Services: Washington, DC, USA, 1985.

15. World Health Organization (WHO). Laboratory Services in Tuberculosis Control. Part III. For the Global Tuberculosis Programme. 1998. Available online: http://wwwn.cdc.gov/dls/ila/documents/lstc3.pdf (accessed on 10 May 2021).

16. Deshpande, P.S.; Kashyap, R.S.; Ramteke, S.S.; Nagdev, K.J.; Purohit, H.J.; Taori, G.M.; Daginawala, H.F. Evaluation of the IS6110 PCR assay for the rapid diagnosis of tuberculous meningitis. Cerebrospin. Fluid Res. 2007, 4, 10. [CrossRef] [PubMed]

17. Jain, S.; Sankar, M.M.; Sharma, N.; Singh, S.; Chugh, T.D. High prevalence of non-tuberculous mycobacterial disease among non-HIV infected individuals in a TB endemic country-Experience from a tertiary center in Delhi, India. Pathog. Glob. Health 2014, 108, 118-122. [CrossRef] [PubMed]

18. Jani, M.N.; Rodrigues, C.S.; Mehta, A.P. The neglected and often ignored: Nontuberculous mycobacteria. J. Glob. Infect. Dis. 2011, 3, 94. [CrossRef] [PubMed] 
19. Cuevas, L.E.; Al-Sonboli, N.; Lawson, L.; Yassin, M.A.; Arbide, I.; Al-Aghbari, n.; Sherchand, J.B.; Al-Absi, A.; Emenyonu, E.N.; Merid, Y.; et al. LED fluorescence microscopy for the diagnosis of pulmonary tuberculosis: A multi-country cross-sectional evaluation. PLoS Med. 2011, 8, e1001057. [CrossRef] [PubMed]

20. Shinu, P.; Nair, A.; Jad, B.; Singh, V. Evaluation of two pretreatment methods for the detection of Mycobacterium tuberculosis in suspected pulmonary tuberculosis. J. Basic Microbiol. 2013, 53, 260-267. [CrossRef] [PubMed]

21. Marei, A.M.; El-Behedy, E.M.; Mohtady, H.A.; Afify, A.F. Evaluation of a rapid bacteriophage-based method for the detection of Mycobacterium tuberculosis in clinical samples. J. Med. Microbiol. 2003, 52, 331-335. [CrossRef] [PubMed]

22. Sankar, M.M.; Kumar, P.; Munawwar, A.; Singh, J.; Parashar, D.; Singh, S. Recovery of Mycobacterium tuberculosis from sputum treated with cetyl pyridinium chloride. J. Clin. Microbiol. 2009, 47, 4189-4190. [CrossRef] [PubMed] 